\title{
Antenatal corticosteroids for maturity of term or near term fetuses: systematic review and meta-analysis of randomized controlled trials
}

\author{
Gabriele Saccone, ${ }^{1}$ Vincenzo Berghella²
}

${ }^{1}$ Department of Neuroscience, Reproductive Sciences and Dentistry, School of Medicine, University of Naples Federico II, Naples, Italy

2Division of Maternal-Fetal Medicine, Department of Obstetrics and Gynecology, Sidney Kimmel Medical College of Thomas Jefferson University, Philadelphia, PA 19107, USA

Correspondence to: $\vee$ Berghella vincenzo.berghella@jefferson.edu Additional material is published online only. To view please visit the journal online.

Cite this as: $B M J$ 2016;355:i5044 http://dx.doi.org/10.1136/bmj.i5044

Accepted: 12 September 2016

\author{
ABSTRACT \\ OBJECTIVE \\ To evaluate the effectiveness of antenatal \\ corticosteroids given at $\geq 34$ weeks' gestation. \\ DESIGN \\ Systematic review with meta-analysis.

\section{DATA SOURCES} \\ Electronic databases were searched from their \\ inception to February 2016.
}

\section{ELIGIBILITY CRITERIA FOR STUDY SELECTION}

Randomized clinical trials comparing antenatal corticosteroids with placebo or no treatment in women with a singleton pregnancy at $\geq 34$ weeks' gestation. Trials on antenatal steroids in women expected to deliver late preterm ( $34^{0}-36^{6}$ weeks) and trials given before planned cesarean delivery at term ( $\geq 37$ weeks) were included.

\section{DATA SYNTHESIS \\ The primary outcome was the incidence of severe respiratory distress syndrome (RDS). The summary measures were reported as relative risks or mean differences with $95 \%$ confidence intervals.}

RESULTS

Six trials, including 5698 singleton pregnancies, were analyzed. Three included 3200 women at $34^{0}-36^{6}$ weeks' gestation and at risk of imminent premature delivery at the time of hospital admission. The three other trials included 2498 women undergoing planned cesarean delivery at $\geq 37$ weeks. Overall, infants of mothers who received antenatal corticosteroids at $\geq 34$ weeks had a significantly lower risk of RDS (relative risk $0.74,95 \%$ confidence interval 0.61 to 0.91$)$, mild $\operatorname{RDS}(0.67,0.46$ to 0.96$)$, moderate $\operatorname{RDS}(0.39,0.18$ to $0.89)$, transient tachypnea of the newborn $(0.56,0.37$ to 0.86$)$, severe $\operatorname{RDS}(0.55,0.33$ to 0.91$)$, use of surfactant, and mechanical ventilation, and a significantly lower time receiving oxygen (mean difference -2.06 hours, $95 \%$ confidence interval -2.17

\section{WHAT IS ALREADY KNOWN ON THIS TOPIC}

Prophylactic corticosteroids in preterm pregnancies accelerate lung maturation and reduce the incidence of respiratory morbidity

Use of antenatal corticosteroids is currently recommended at 24-33 weeks' gestation in women at risk of preterm birth, whereas the evidence for use at or beyond 34 weeks is still debatable

\section{WHAT THIS STUDY ADDS}

Use of antenatal steroids at 34 or more weeks' gestation reduces neonatal respiratory morbidity

A single course of corticosteroids can be considered for women at risk of imminent late premature delivery, as well as for those undergoing planned cesarean at term to -1.95), lower maximum inspired oxygen concentration $(-0.66 \%,-0.69 \%$ to $-0.63 \%)$, shorter stay on a neonatal intensive care unit ( -7.64 days, -7.65 to -7.64 ), and higher APGAR scores compared with controls. Infants of mothers who received antenatal betamethasone at $34^{0}-36^{6}$ weeks' gestation had a significantly lower incidence of transient tachypnea of the newborn (relative risk $0.72,95 \%$ confidence interval 0.56 to 0.92$)$, severe RDS (0.60, 0.33 to 0.94$)$, and use of surfactant $(0.61,0.38$ to 0.99). Infants of mothers undergoing planned cesarean delivery at $\geq 37$ weeks' gestation who received prophylactic antenatal corticosteroids 48 hours before delivery had a significantly lower risk of $\operatorname{RDS}(0.40,0.27$ to 0.59$)$, mild $\operatorname{RDS}(0.43,0.26$ to 0.72$)$, moderate RDS $(0.40,0.18$ to 0.88$)$, transient tachypnea of the newborn $(0.38,0.25$ to 0.57$)$, and mechanical ventilation $(0.19,0.08$ to 0.43$)$, and significantly less time receiving oxygen (mean difference -2.06 hours, 95\% confidence interval -2.17 to -1.95), lower percentage of maximum inspired oxygen concentration $(-0.66 \%,-0.69 \%$ to $-0.63 \%)$, shorter stay in neonatal intensive care $(-7.44$ days, -7.44 to -7.43 ), and a higher APGAR score at one and at five minutes.

\section{CONCLUSIONS}

Antenatal steroids at $\geq 34$ weeks' gestation reduce neonatal respiratory morbidity. A single course of corticosteroids can be considered for women at risk of imminent late premature delivery $34^{0}-36^{6}$ weeks' gestation, as well as for women undergoing planned cesarean delivery at $\geq 37$ weeks' gestation.

\section{SYSTEMATIC REVIEW REGISTRATION} PROSPERO CRD42016035234.

\section{Introduction}

Respiratory distress syndrome (RDS) is one of the most important causes of early neonatal morbidity and mortality. ${ }^{1}$ In the United States it affects about $20 \%$ of low birthweight infants and 30\% of extremely low birthweight infants. ${ }^{1}$ Infants born at late preterm, between 34 and 36 weeks' gestation, are more likely to experience respiratory complications than infants born at term. Cesarean delivery is also a risk factor for the development of neonatal respiratory complications, including RDS. ${ }^{12}$

Respiratory failure in these babies occurs as a result of surfactant deficiency, poor anatomical development of lung, as well as immaturity in other organs. ${ }^{2}$ Neonatal survival after preterm birth improves with length of gestation, reflecting improved maturity of organ systems. ${ }^{2}$ 
Prophylactic corticosteroids in singleton preterm pregnancies accelerate lung maturation and reduce the incidence of RDS. ${ }^{3}$ Therefore, antenatal corticosteroids are currently recommended between 24 and $33^{6}$ weeks' gestation in women at risk of preterm birth within seven days-for example, in cases of threatened preterm labor, preterm premature rupture of membranes, antepartum hemorrhage. ${ }^{45}$ The evidence for use of antenatal steroids at or after 34 weeks is still debatable. ${ }^{3-5}$ Recently, the American College of Obstetricians and Gynecologists recommended antenatal corticosteroids for women at risk of late premature delivery at greater than 34 weeks' gestation but not for women undergoing planned cesarean at term; ${ }^{5}$ whereas according to the Royal College of Obstetricians and Gynaecologists antenatal corticosteroids should be given to all women with a planned elective cesarean section prior to $38^{6}$ weeks' gestation. ${ }^{4}$

In this systematic review and meta-analysis we evaluated the effectiveness of antenatal corticosteroids given at or after 34 weeks to reduce neonatal respiratory morbidity.

\section{Methods}

\section{Search strategy and selection criteria}

This review was performed according to a protocol designed a priori and recommended for systematic reviews. ${ }^{6}$ We searched Medline, Scopus, ClinicalTrials. gov, Embase, Sciencedirect, the Cochrane Library at the CENTRAL Register of Controlled Trials, Scielo from their inception to February 2016. Search terms used were: “cesarean”, “caesarean”, "steroids”, “corticosteroids”, “planned”, “elective”, “term”, “preterm”, “infants”, "respiratory”, "morbidity”, “mortality”, "meta-analysis", "metaanalysis", "review”, "randomized”, "randomised", "effectiveness", "antenatal", "disorders", "late", and "clinical trial". No restrictions for language or geographic location were applied. In addition, we examined the reference lists of all identified articles to find studies not captured by electronic searches. Two authors (GS, VB) independently assessed the electronic search and the eligibility of the studies. Differences were discussed and consensus reached.

We included all randomized controlled trials comparing use of antenatal corticosteroids (intervention group) with placebo or no treatment (control group) in women with a singleton pregnancy at 34 or more weeks. We also included trials on use of antenatal steroids in both women expected to deliver in late preterm $\left(34^{0}-36^{6}\right.$ weeks) and women before planned cesarean delivery at term ( $\geq 37$ weeks). Trials on prophylactic antenatal corticosteroids given at less than 34 weeks and those including multiple pregnancies and quasi-randomized trials (trials with allocation done on the basis of a pseudo-random sequence, such as odd or even hospital number or date of birth, alternation) were also excluded.

Data extraction and risk of bias assessment To assess the risk of bias in each included study we used the criteria outlined in the Cochrane Handbook. ${ }^{6}$
In each included trial we assessed seven domains related to risk of bias, as evidence suggests that these are associated with biased estimates of treatment effect: random sequence generation, allocation concealment, blinding of participants and staff, blinding of outcome assessment, incomplete outcome data, selective reporting, and other bias. The review authors categorized studies as "low risk," "high risk," or "unclear risk" of bias. ${ }^{6}$

Two authors (GS, VB) independently assessed inclusion criteria, risk of bias, and data extraction. Disagreements were resolved by discussion.

We carried out all analyses using an intention to treat approach, evaluating women according to the treatment group to which they were randomly allocated in the original trials. Primary and secondary outcomes were defined before data extraction. The primary outcome was the incidence of severe RDS. ${ }^{7}$ Secondary outcomes were incidence of overall RDS (mild, moderate, or severe) ${ }^{7}$ incidence of mild and moderate RDS; ${ }^{7}$ incidence of transient tachypnea of the newborn; ${ }^{7}$ use of surfactant, mask ventilation, and mechanical ventilation; time receiving oxygen (hours); maximum inspired oxygen concentration (\%); incidence of admission to neonatal intensive care unit; length of stay in the neonatal intensive care unit (days); APGAR score at one and at five minutes; neonatal hypoglycemia (glucose $<2.2 \mathrm{mmol} / \mathrm{L}$ ); and neonatal mortality (defined as death of a liveborn baby within the first 28 days of life).

We separately assessed the primary and secondary outcomes in the main analysis as well as in studies on antenatal steroids in women expected to deliver late preterm ( $34^{0}-36^{6}$ weeks) and in studies on antenatal steroids given immediately before scheduled cesarean delivery at 37 or more weeks. We also planned to assess the primary outcome (incidence of severe RDS) in sensitivity analysis according to the study design of the original trial, excluding studies without placebo as control.

\section{Statistical analysis}

Two authors (GS, VB) independently completed the data analysis using Review Manager v. 5.3. They compared the completed analyses, and resolved any differences by discussion.

Using custom-made data collection forms, we extracted data from each eligible study without modification of the original data. A $2 \times 2$ table was assessed for relative risk; for continuous outcomes means with or without standard deviations were extracted and imported into Review Manager v. 5.3.

Meta-analysis was performed using the random effects model of DerSimonian and Laird, to produce summary treatment effects in terms of either relative risks or mean differences, with $95 \%$ confidence intervals. Heterogeneity was measured using the $\mathrm{I}^{2}$ statistic (Higgins $\mathrm{I}^{2}$ ). To account for the uncertainty in estimating heterogeneity, we converted DerSimonian and Laird results to Hartung and Knapp results, which accounts for the extra uncertainty. ${ }^{8}$

For individual trials with no events in one or both groups, we added a continuity correction of 0.5 to each cell for each effect measure. ${ }^{6}$ 
Potential publication biases were assessed graphically by using a funnel plot of the primary outcome and statistically by using Begg's and Egger's tests. We considered a P value of less than 0.05 to be significant.

The meta-analysis was reported following the preferred reporting item for systematic reviews and meta-analyses (PRISMA) statement. ${ }^{9}$ Before data extraction, we registered the review with PROSPERO following the PRISMA guidelines for protocols (registration number CRD42016035234). ${ }^{10}$

\section{Patient involvement}

No patients were involved in setting the research question or the outcome measures, nor were they involved in developing plans for design, or implementation of the study. No patients were asked to advise on interpretation or writing up of results. There are no plans to disseminate the results of the research to study participants or the relevant patient community.

\section{Results}

Figure 1 shows the flow of study identification. Supplementary file S1 details the full electronic search. Six trials, including 5698 singleton pregnancies, were identified as relevant and included in the metaanalysis. ${ }^{11-16}$ Data were also obtained by a follow-up of a randomized controlled trial. ${ }^{17}$ Publication bias could not be assessed for all the different outcomes, given the small $(<10)$ number of studies included.

Most of the included studies (four out of the six), ${ }^{1315}$ were judged as low risk of bias in most of the seven Cochrane domains related to the risk of bias. All the included studies had low risk of bias in random sequence generation. Adequate methods for allocation of women were used in all the included trials, except two in which details on methods used to conceal allocation were not reported. ${ }^{1316}$ In three double blind studies, ${ }^{12} 1416$ neither the participants nor the investigators were aware of the treatment assignments. Three trials were judged as low risk of bias for incomplete outcome data, ${ }^{11214}$ two as unclear, ${ }^{1315}$ and one as high risk ${ }^{16}$ (figs 2 and 3). Supplementary file S2 shows the details of the risk of bias assessment for each included study.

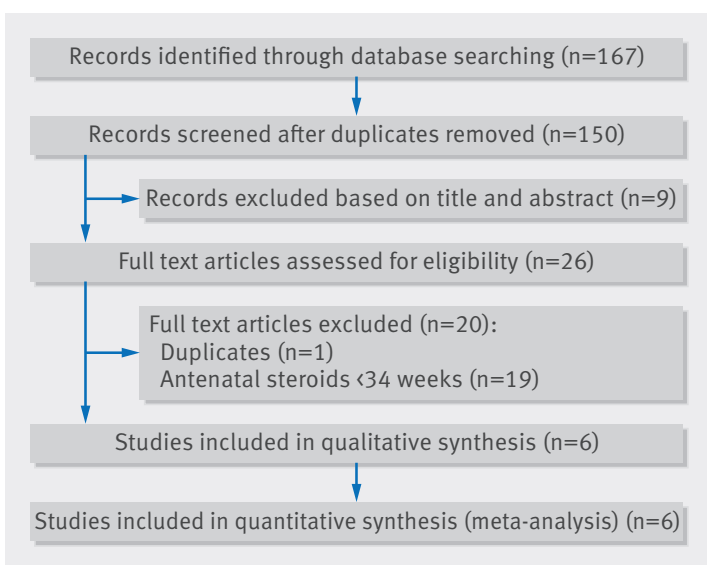

Fig 1 | Flow diagram of studies identified in systematic review
Table 1 shows the characteristics of the included clinical trials. All six studies excluded multiple pregnancies. Four trials used betamethasone as corticosteroid ${ }^{11121415}$ and two used dexamethasone. ${ }^{1316}$ Three trials used placebo as control. ${ }^{111416}$ In all of the included studies but two, ${ }^{1516}$ corticosteroids were given intramuscularly in two doses of $12 \mathrm{mg}$ separated by 24 hours. The first trial used a single dose of $12 \mathrm{mg}$ antenatal betamethasone. ${ }^{15}$ In the other trial, the intervention group consisted of 645 women who received intramuscular dexamethasone $8 \mathrm{mg}$ every 12 hours for two days, 48 hours before planned cesarean delivery. ${ }^{16}$ None of the trials assessed compliance to the treatment.

Three trials included 2498 women undergoing planned cesarean delivery at 37 or more weeks. ${ }^{11316}$ The other three studies included 3200 women at $34^{0}-36^{6}$ weeks' gestation and at risk of imminent late premature delivery (spontaneously or as indicated) at the time of admission to hospital. ${ }^{121415}$ High probability of delivery in late preterm period ( $34^{0}-36^{6}$ weeks) included any one of the following: membrane rupture; preterm labor with intact membranes, defined as at least six regular uterine contractions in an observation period of no more than 60 minutes and cervix either greater than or equal to $3 \mathrm{~cm}$ dilated or $80 \%$ effaced; planned delivery by induction of labor or cesarean scheduled by $36^{6}$ weeks' gestation at the latest for any indication, including prior myomectomy, prior classic cesarean, intrauterine growth restriction, pre-eclampsia, oligohydramnios, non-reassuring fetal heart rate tracing warranting delivery, placental abruption, or placenta previa. The US trial excluded women with pregestational diabetes

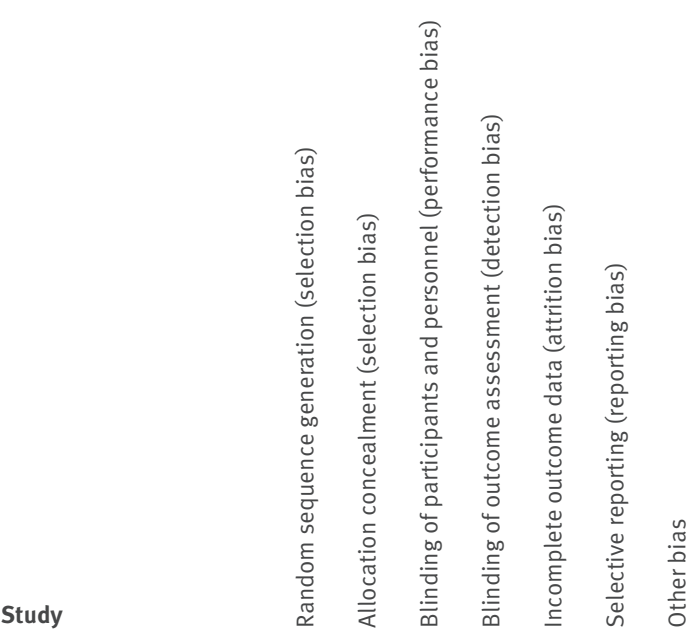

Ahmed 2015

Balci 2010

Gyamfi-Bannerman 2016

Nada 2016

Porto 2011

Stutchfield 2005

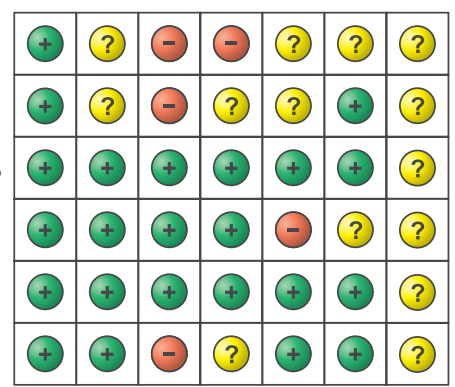

Fig 2 | Summary of risk of bias for each trial. Green=low risk of bias; red=high risk of bias; yellow=unclear risk of bias 


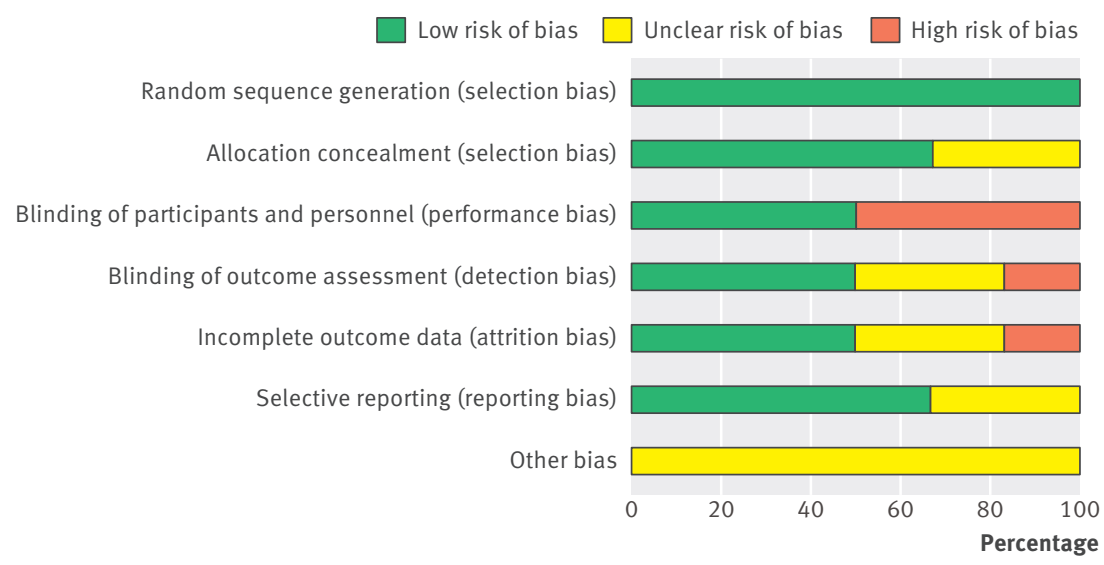

Fig 3 Risk of bias item presented as percentages across all included studies. Green=low risk of bias; red=high risk of bias; yellow=unclear risk of bias

and women who previously received a course of corticosteroids. Tocolysis was not employed as part of this trial, and delivery was not delayed for obstetric or medical indications. ${ }^{14}$

RDS was defined in the studies as the presence of clinical signs of respiratory distress with an oxygen requirement and a chest radiograph showing reticulogranular infiltrates. ${ }^{7}$ Transient tachypnea of the newborn was defined as respiratory distress without diffuse reticulogranular infiltrate resolved spontaneously by 72 hours of age. ${ }^{7}$

In three trials, incidence of RDS was the primary outcome; $;^{121315}$ in two trials, incidence of admission to a neonatal intensive care unit was the primary outcome; ${ }^{116}$ whereas the US trial defined the primary outcome as a composite endpoint describing the need for respiratory support by 72 hours of age and consisted of one or more of the following: continuous positive airway pressure or high flow nasal cannula for at least two consecutive hours, an oxygen requirement with a fraction of inspired oxygen of at least 30\% for at least four continuous hours, extracorporeal membrane oxygenation, or the need for mechanical ventilation. ${ }^{14}$

Table 2 shows primary and secondary outcomes in the main analysis. Women who received prophylactic antenatal corticosteroids (betamethasone or dexamethasone) after 34 weeks had a significantly lower incidence of RDS (relative risk $0.74,95 \%$ confidence interval 0.61 to 0.91; fig 4), mild RDS (0.67, 0.46 to 0.96$)$, moderate RDS $(0.39,0.18$ to 0.89$)$, transient tachypnea of the newborn (0.56, 0.37 to 0.86 ; fig 5), severe RDS ( $0.55,0.33$ to 0.91 ; fig 6), use of surfactant (0.61, 0.38 to 0.99$)$, use of mechanical ventilation $(0.52,0.36$ to 0.76$)$, significantly lower time receiving oxygen (mean difference-2.06 hours, 95\% confidence interval -2.17 to -1.95$)$, lower maximum inspired oxygen concentration $(-0.66 \%,-0.69$ to -0.63$)$, lower length of stay in the neonatal intensive care unit (-7.64 days, -7.65 to -7.64$)$, higher APGAR score at one and at five minutes $(0.06,0.05$ to 0.07$)$, and higher neonatal hypoglycemia (1.61, 1.38 to 1.87 ) compared with those who did not. After converting results using the Hartung and Knapp approach, use of surfactant was no longer significant. No statistically significant differences were found in use of mask ventilation (relative risk 0.85, $95 \%$ confidence interval 0.69 to 1.04 ), admission to a neonatal intensive care unit (0.81, 0.54 to 1.20$)$, and neonatal death $(0.77,0.21$ to 2.83$)$.

Table 3 shows the primary and secondary outcomes in women at risk of late premature delivery $\left(34^{0}-36^{6}\right.$ weeks). Women who received antenatal betamethasone at $34^{\circ}$ $36^{6}$ weeks' gestation had a significantly lower incidence of transient tachypnea of the newborn (0.72, 0.56 to $0.92)$, severe RDS (0.60, 0.33 to 0.94$)$, and use of surfactant $(0.61,0.38$ to 0.99 ,) but higher neonatal hypoglycemia (1.61, 1.38 to 1.87$)$. After converting results using the Hartung and Knapp approach, use of surfactant was no longer significant. No statistically significant differences were found in the incidence of overall $\operatorname{RDS}(0.98,0.77$ to 1.24), mild RDS (1.09, 0.66 to 1.80$)$, mask ventilation (0.83, 0.67 to 1.02$)$, mechanical ventilation $(0.80,0.51$ to $1.24)$, admission to a neonatal intensive care unit (0.94, 0.87 to 1.02), APGAR score at one minute (mean difference $-0.46,95 \%$ confidence interval -0.78 to 0.14 ) and at five minutes $(-0.62,-0.91$ to 0.33$)$, and neonatal death (relative risk $0.95,95 \%$ confidence interval 0.20 to 4.58 ).

In sensitivity analysis according to study design of the included trials, excluding one study, ${ }^{15}$ we found that women at risk of late premature delivery who received antenatal corticosteroids at 34 or more weeks still had a significantly lower risk of the primary outcome (incidence of severe RDS) in the pooled data from only trials that used placebo as control $(0.67,0.54$ to 0.84; table 4). ${ }^{1214}$

Table 5 shows the primary and secondary outcomes in women undergoing scheduled cesarean delivery at term. Women undergoing planned cesarean delivery at $37^{\circ}$ or more weeks who received prophylactic antenatal corticosteroids (betamethasone or dexamethasone) 48 hours before delivery had a significantly lower risk of RDS (0.40, 0.27 to 0.59), mild RDS (0.43, 0.26 to 0.72 ), moderate RDS (0.40, 0.18 to 0.88), transient tachypnea of the newborn $(0.38,0.25$ to 0.57$)$, mechanical ventilation (0.19, 0.08 to 0.43$)$, significantly lower time receiving oxygen (mean difference -2.06 hours, 95\% confidence interval -2.17 to -1.95$)$, lower percentage of maximum inspired oxygen concentration $(-0.66 \%$, -0.69 to -0.63$)$, lower length of stay in the neonatal intensive care unit ( -7.44 days, -7.44 to -7.43$)$, and higher APGAR scores at one and five minutes (0.06, 0.07 to 0.05 ). We found no statistically significant differences in number of fetuses who required mask ventilation (relative risk 1.06, 95\% confidence interval 0.53 to 2.08), in the incidence of admission to the neonatal intensive care unit $(0.48,0.19$ to 1.20$)$, and in the incidence of neonatal death (0.50, 0.05 to 5.46$)$.

\section{Discussion}

This meta-analysis of the findings from six randomized controlled trials, ${ }^{11-16}$ including 5698 singleton pregnancies, evaluated the efficacy of prophylactic antenatal corticosteroids (betamethasone or dexamethasone) given at or after 34 weeks. Our findings showed that antenatal steroids administered at 34 or more weeks' gestation reduce neonatal respiratory morbidity. 


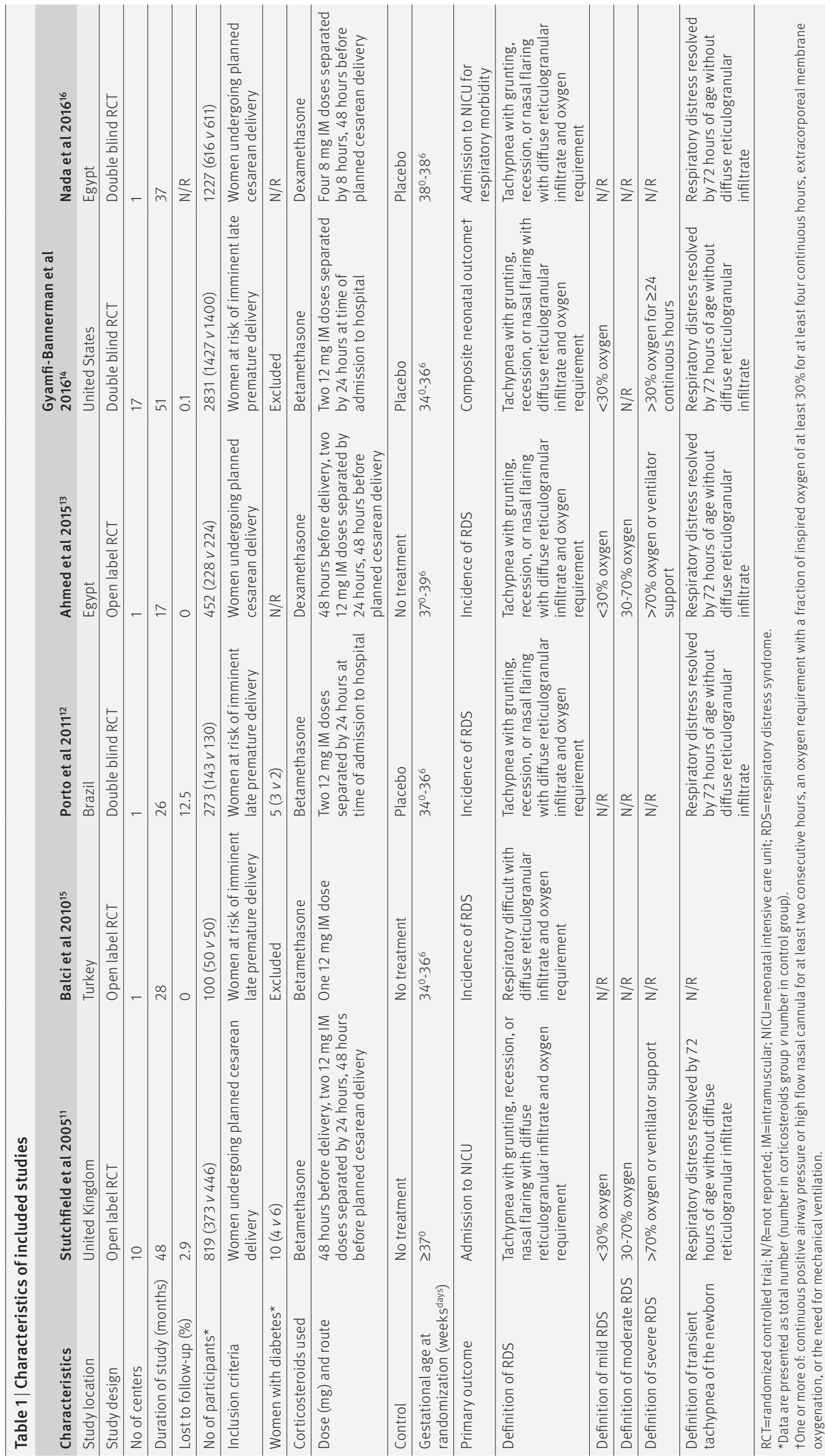




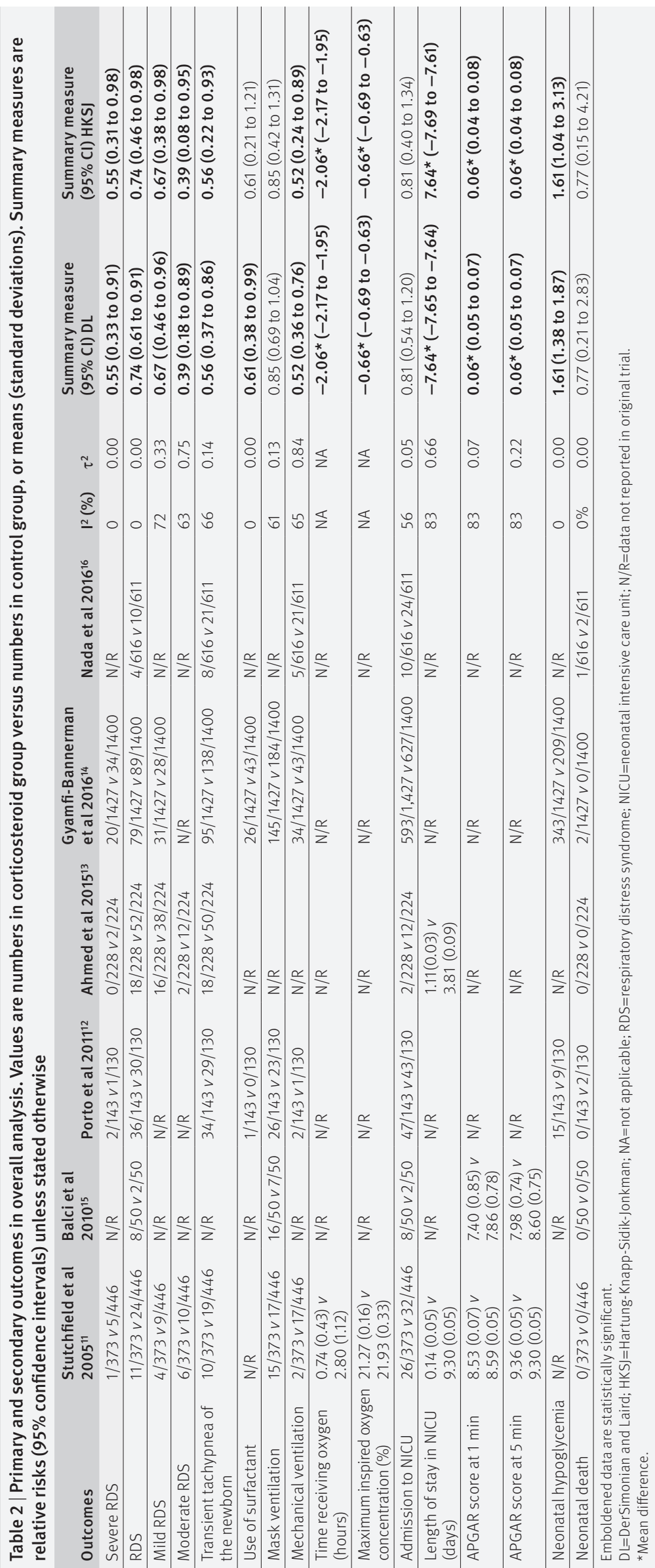

Our meta-analysis showed neonatal benefit of antenatal betamethasone in women at immediate risk of late preterm delivery $\left(34^{0}-36^{6}\right.$ weeks). A single course of antenatal corticosteroids given immediately before planned cesarean delivery at 37 or more weeks' gestation was also associated with a reduction in the rates of mild, moderate, and severe respiratory distress syndrome (RDS). Out of the three trials included, one enrolled women undergoing planned cesarean delivery at $37^{0}-39^{6}$ weeks' gestation, ${ }^{13}$ one enrolled women at 37 weeks or beyond, ${ }^{11}$ and one enrolled women at $38^{0}-38^{6}$ weeks. ${ }^{16}$ Another study also found that the rate of RDS decreased with increasing gestation, supporting the recommendation to delay planned cesarean delivery until the 39th week. ${ }^{11}$

\section{Comparison with other studies}

Two current Cochrane reviews have reported data on the effects of antenatal corticosteroids. ${ }^{318}$ The first meta-analysis aimed to assess the effects on fetal and neonatal morbidity and mortality of administering corticosteroids to the mother before anticipated preterm delivery. ${ }^{3}$ The evidence from this meta-analysis supported the continued use of a single course of antenatal corticosteroids to accelerate fetal lung maturation in women at risk of preterm birth of less than 34 weeks' gestation. However they did not evaluate the effectiveness of antenatal corticosteroids given at or after 34 weeks. $^{3}$ The second meta-analysis, ${ }^{18}$ including only one trial (942 participants), ${ }^{13}$ showed that antenatal steroids did not prevent RDS compared with no treatment in women undergoing planned cesarean delivery at 37 or more weeks' gestation.

\section{Strengths and limitations of this review}

Our study has several strengths. The six trials included had a low risk of allocation bias by Cochrane Collaboration tool assessment. An intention to treat analysis was used. To our knowledge, no prior meta-analysis on this issue is as large, up to date, or comprehensive.

Limitations of our study are mostly inherent to the limitations of the included studies. Only three trials used placebo as control and were double blind. Data on optimal dose to delivery interval and optimal corticosteroid to use were limited. Almost half of the women included in the analysis (2831 out of the 5698) came from one large well designed trial. ${ }^{14}$ Performing subgroup analysis by mode of delivery in the late preterm group was not feasible. The major shortcoming was the lack of data on long term outcomes. Only one trial reported long term follow-up, ${ }^{17}$ so the long term safety of antenatal corticosteroids at late preterm or term is still not well known. One trial reported that antenatal betamethasone did not result in any adverse long term neurological or cognitive outcomes at age $8-15$ years. ${ }^{17}$

\section{Interpretation of the findings}

Dexamethasone and betamethasone are the two antenatal corticosteroids recommended for accelerating fetal lung development in threatened preterm birth. ${ }^{3-5}$ The American College of Obstetricians and 


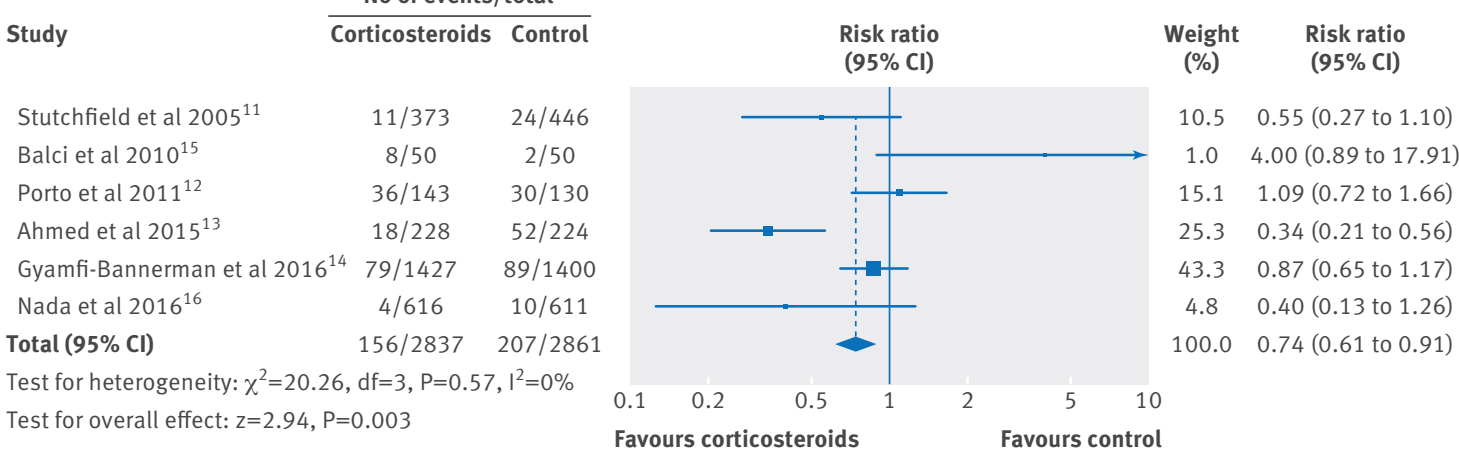

Fig 4 | Forest plot for use of antenatal corticosteroids after 34 weeks' gestation and risk of respiratory distress syndrome

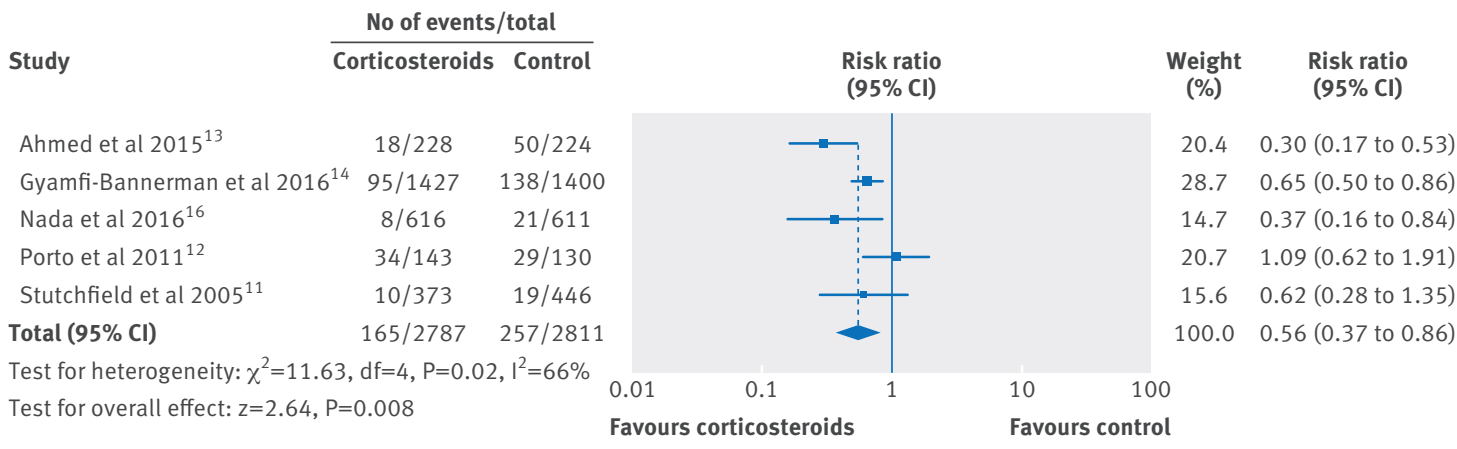

Fig 5 | Forest plot for use of antenatal corticosteroids after 34 weeks' gestation and risk of transient tachypnea of the newborn

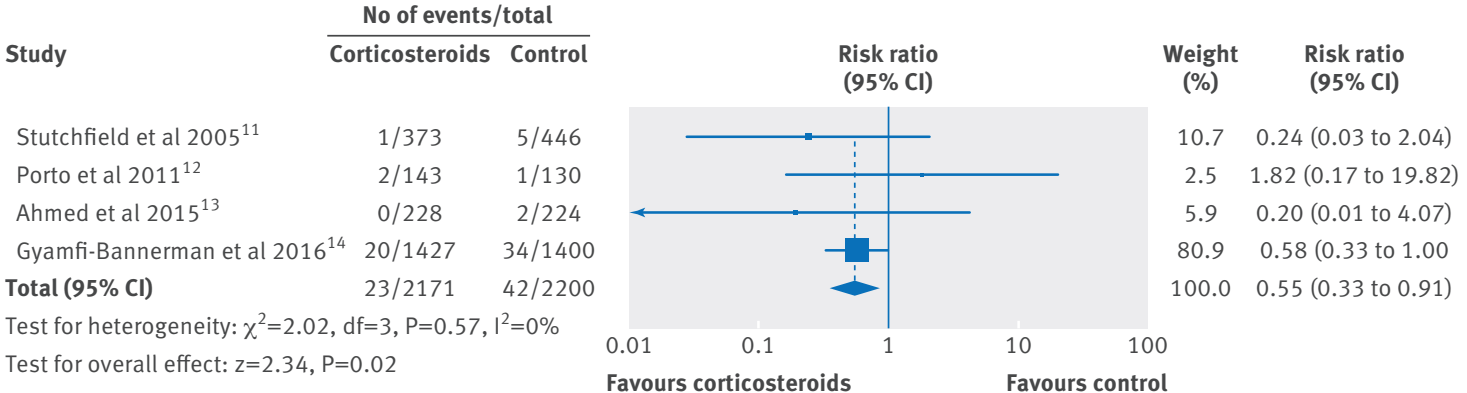

Fig 6 | Forest plot for use of antenatal corticosteroids after 34 weeks' gestation and risk of severe respiratory distress syndrome

Gynecologists and Royal College of Obstetricians and Gynaecologists list both as effective drugs for preventing complications of prematurity, using either a dosage of $24 \mathrm{mg}$ dexamethasone (four $6 \mathrm{mg}$ doses 12 hours apart) or $24 \mathrm{mg}$ betamethasone (two $12 \mathrm{mg}$ doses 24 hours apart). ${ }^{45}$ In a 2013 Cochrane Review, evaluating the effects of different corticosteroid regimens for women at risk of preterm birth, the reviewers concluded that "It remains unclear whether one corticosteroid (or one particular regimen) has advantages over another."19

A single course of antenatal corticosteroids should be considered routine for women at less than 34 weeks' gestation for risk of preterm birth, ${ }^{3}$ whereas so far evidence for administering antenatal steroids at or after 34 weeks is still debatable. Our meta-analysis based on level 1 data showed that antenatal corticosteroids after 34 weeks' gestation improved neonatal outcomes.
The biological plausibility to explain our findings may be different in women undergoing scheduled cesarean delivery at term and in women at risk of late preterm delivery.

Cesarean delivery is a risk factor for the development of neonatal respiratory complications, mostly RDS and transient tachypnea of the newborn, in infants both at term and preterm. ${ }^{2021}$ Infants born at term by cesarean delivery are more likely to develop respiratory morbidity than infants born vaginally, and this risk increases further for the subgroup of children born after scheduled cesarean section-that is, before onset of labor with potentially severe implications. The risk decreases with advancing gestational age, and infants born at $37^{0}-37^{6}$ weeks' gestation are at 1.7 times more risk for respiratory complications than those born at $38^{0}-38^{6}$ weeks' gestation who in turn are at 2.4 times more risk 


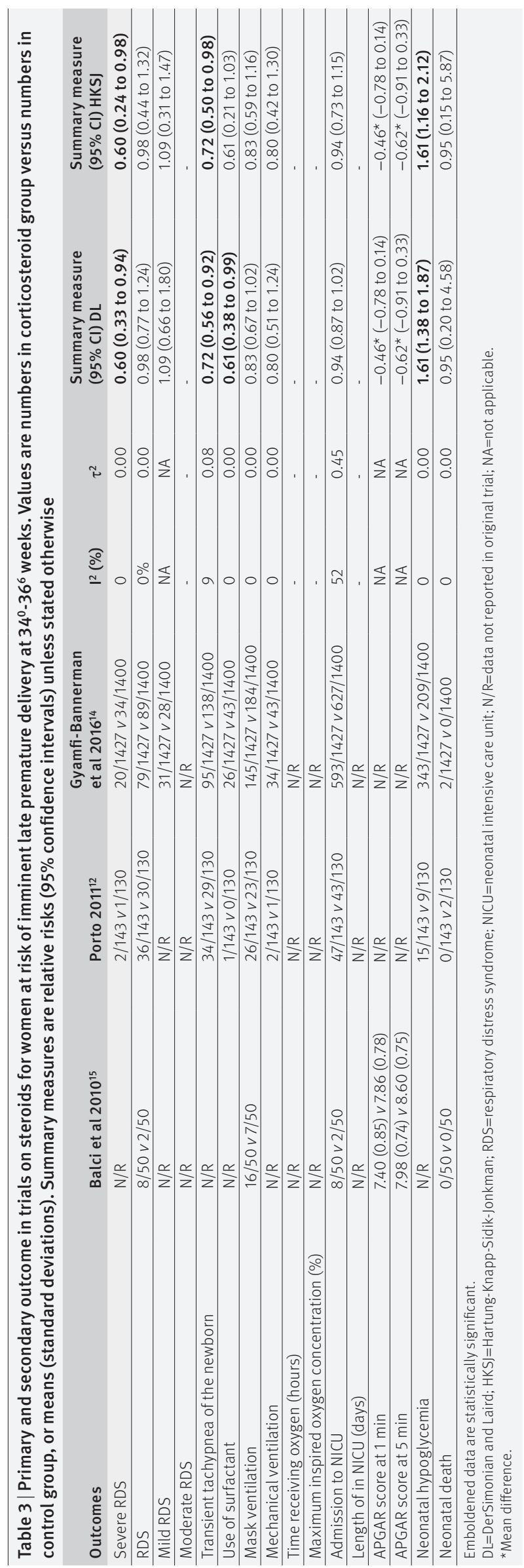

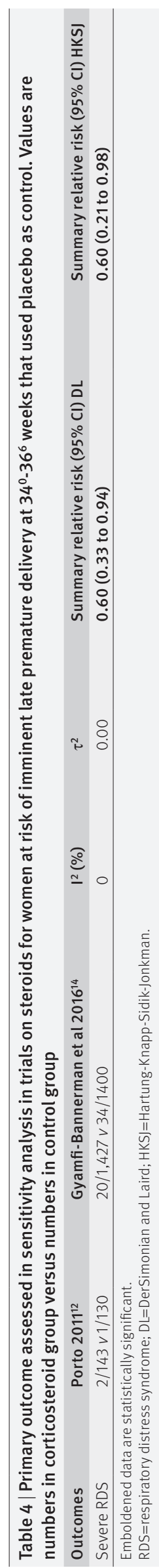

than the infants born at $39^{0}-39^{6}$ weeks' gestation. ${ }^{20}$ This trend is particularly pronounced for RDS. ${ }^{21}$ Respiratory morbidity in term planned cesarean delivery seems to have a different pathophysiology from that in preterm birth, with lack of the physiological catecholamine surge ${ }^{2223}$ and fluid retention in the lungs ${ }^{2425}$ being the most likely causes. ${ }^{22-25}$ Interestingly, recent evidence indicates that, apart from the mechanical concept of vaginal squeeze, molecular mechanisms (lung epithelial sodium channels) promote alveolar fluid drainage, and these channels may be underactive in fetuses not exposed to the process of labor. ${ }^{22-25}$ Glucocorticoids appear to increase the number and function of sodium channels as well as the responsiveness to catecholamine and thyroids hormones, providing a rationale for their exogenous use in planned cesarean deliveries. ${ }^{22-25}$ In view of this evidence, it is currently recommended that planned cesarean delivery should be deferred to 39 weeks' gestation. ${ }^{2627}$ However, in the USA approximately $10-15 \%$ of women planned for cesarean might deliver before 39 weeks and there may be concerns on waiting in the presence of specific clinical indications or history. ${ }^{1}$ Some have referred to planned cesarean delivery as "elective," but we prefer avoiding this term, as it lacks the necessary scientific specificity. ${ }^{28}$ The American College of Obstetricians and Gynaecologists advises against “elective delivery” before 39 weeks' gestation. ${ }^{23}$ The challenge in this situation is that there is often lack of clarity between planned (scheduled) delivery and an elective delivery. Cesarean delivery scheduled at 35 weeks' gestation because of placenta previa and suspected accreta is not an "elective" early preterm birth, and an increase in maternal-fetal morbidity has been associated with allowing such a pregnancy to continue beyond 36 weeks' gestation. ${ }^{29}$

Infants born at late preterm, between 34 and 36 weeks' gestation, are more likely to experience respiratory complications than infants born at term..$^{30}$ Our findings suggested that antenatal betamethasone in women at immediate risk of late preterm delivery $\left(34^{0}-36^{6}\right.$ weeks) improves neonatal outcomes.

Notably, our meta-analysis also showed an increased risk of neonatal hypoglycemia, in women who were randomized to antenatal corticosteroids at more than 34 weeks' gestation. Therefore, an important question is whether the short term benefits showed by this meta-analysis might be associated with any long term benefits or risks. In this context, the increase in the incidence of neonatal hypoglycemia is particularly worrisome, especially since neonatal hypoglycemia has been reported to be the only independent risk factor for later developmental delay and physical growth deficits among moderate or late preterm infants. ${ }^{31}$ However, no adverse events related to hypoglycemia were reported in any trials and the rates of hypoglycemia found are similar to what is reported in the general population of late preterm infants. ${ }^{1431}$

\section{Conclusions}

Prophylactic antenatal corticosteroids given immediately before planned cesarean delivery at 37 or more 


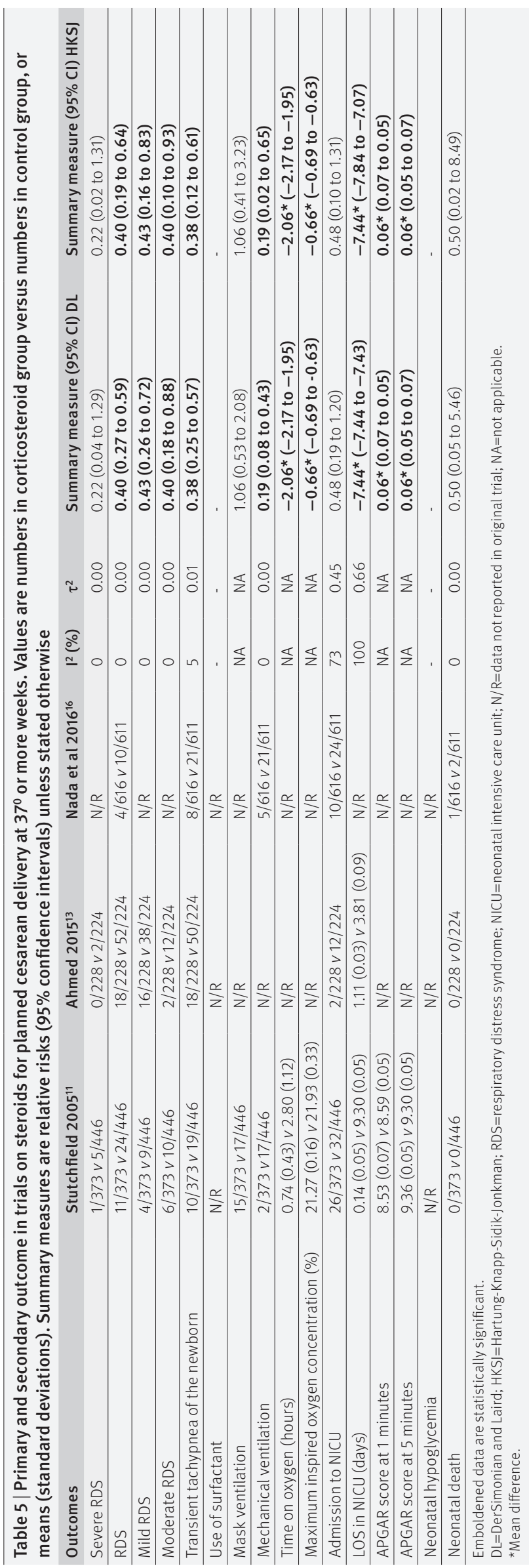

weeks' gestation are effective in reducing RDS. Therefore, in planned cesareans of infants at term, the risk of respiratory morbidity should be considered and the likely benefits of antenatal steroids compared with those of delaying delivery until 39 weeks' gestation when possible. Delivery by scheduled cesarean delivery should be delayed until the 39th week whenever possible to reduce the risk of respiratory morbidity. When it is necessary to deliver by prelabor at $37^{0}-38^{6}$ weeks' gestation, parents can be counseled about the benefits of a single course of antenatal corticosteroids, such as a reduction in RDS from $6.7 \%$ (86/1281) to $2.7 \%$ (33/1217) (table 5).

Prophylactic betamethasone is also beneficial in decreasing neonatal respiratory morbidities (such as severe RDS, from 2.3\% (35/1530) to 1.4\% (22/1570) (table 3)) in women at risk of imminent late premature delivery (34 $4^{0}-36^{6}$ weeks' gestation). Tocolysis should not be used in order to delay delivery to allow for administration of late preterm antenatal corticosteroids, nor should an indicated late preterm delivery (such as for pre-eclampsia with severe features) be postponed for steroid administration.

In conclusion, a single course of corticosteroids (either two $12 \mathrm{mg}$ doses of betamethasone given intramuscularly 24 hours apart or four $6 \mathrm{mg}$ doses of dexamethasone administered intramuscularly every 12 hours) should be considered for women undergoing planned cesarean at 37 or more weeks' gestation as well as for women at risk of imminent late premature delivery at $34^{0}-36^{6}$ weeks' gestation.

Further studies are required on the optimal dose to delivery interval, optimal corticosteroid to use, effects in multiple pregnancies, long term effects into adulthood, as well as in groups not well studied, including women with pregestational diabetes and those who previously had received a course of corticosteroids.

Contributors: VB conceived and designed the study. Both authors acquired, analysed, or interpreted the data; carried out the statistical analysis, provided administrative, technical, or material support, and cirtically revised the manuscript. VB supervised the study and is the guarantor.

Funding: This study received no financial support.

Competing interests: All authors have completed the ICMJE uniform disclosure form at http://www.icmje.org/coi_disclosure.pdf and declare: no support from any organization for the submitted work; no financial relationships with any organizations that might have an interest in the submitted work in the previous three years; no other relationships or activities that could appear to have influenced the submitted work.

Ethical approval: Not required.

\section{Data sharing: No additional data available.}

Transparency: The lead author (VB) affirms that the manuscript is an honest, accurate, and transparent account of the study being reported; that no important aspects of the study have been omitted; and that any discrepancies from the study as planned have been explained.

This is an Open Access article distributed in accordance with the Creative Commons Attribution Non Commercial (CC BY-NC 3.0) license, which permits others to distribute, remix, adapt, build upon this work non-commercially, and license their derivative works on different terms, provided the original work is properly cited and the use is noncommercial. See: http://creativecommons.org/licenses/by-nc/3.0/.

Martin JA, Hamilton BE, Osterman MJ, Curtin SC, Matthews TJ. Births: final data for 2013. Natl Vital Stat Rep 2015:64:1-65.

2 Doyle LW. Victorian Infant Collaborative Study Group. Outcome at 5 years of age of children 23 to 27 weeks' gestation: refining the prognosis. Pediatrics 2001;108:134-41. doi:10.1542/peds.108.1.134. 
3 Roberts D, Dalziel S. Antenatal corticosteroids for accelerating fetal lung maturation for women at risk of preterm birth. Cochrane Database Syst Rev 2006;19:CD004454.

4 Royal College of Obstetricians and Gynaecologists (RCOG). Antenatal corticosteroids to prevent respiratory distress syndrome. Clinical Green Top Guidelines. Royal College of Obstetricians and Gynaecologists, 2004.

5 Committee Opinion No. 677. ACOG committee opinion: antenatal corticosteroids therapy for fetal maturation. Obstet Gynecol 2016;128:e187-94. doi:10.1097/AOG.0000000000001715.

6 Higgins JPT, Green S, eds. Cochrane handbook for systematic reviews of interventions, version 5.1.0 (update March 2011). The Cochrane Collaboration, 2011. Available at: www.cochrane-handbook.org. (Accessed Nov 20, 2015)

7 Report of working group of the British Association of Perinata Medicine and Neonatal Nurses Association on categories of babies requiring neonatal care. Arch Dis Child 1992;67:868-9. doi:10.1136/ adc.67.7 Spec No.868.

8 Hartung J, Knapp G. A refined method for the meta-analysis of controlled clinical trials with binary outcome. Stat Med 2001;20:387589. doi:10.1002/sim.1009.

9 Moher D, Liberati A, Tetzlaff J, Altman DG. PRISMA Group. Preferred reporting items for systematic reviews and meta-analyses: the PRISMA statement. J Clin Epidemiol 2009;62:1006-12. doi:10.1016/j. jclinepi.2009.06.005.

10 Shamseer L, Moher D, Clarke M, et al. PRISMA-P Group. Preferred reporting items for systematic review and meta-analysis protocols (PRISMA-P) 2015: elaboration and explanation. BMJ 2015;349:97647. doi:10.1136/bmj.g7647.

11 Stutchfield P, Whitaker R, Russell I. Antenatal betamethasone and incidence of neonatal respiratory distress after elective caesarean section: pragmatic randomised trial. BMJ 2005;331:662-8. doi:10.1136/bmj.38547.416493.06.

12 Porto AM, Coutinho IC, Correia JB, Amorim MM. Effectiveness of antenatal corticosteroids in reducing respiratory disorders in late preterm infants: randomised clinical trial. BMJ 2011;342:d1696. doi:10.1136/bmj.d1696.

13 Ahmed MR, Sayed Ahmed WA, Mohammed TY. Antenatal steroids at 37 weeks, does it reduce neonatal respiratory morbidity? A randomized trial. J Matern Fetal Neonatal Med 2015;28:1486-90 doi:10.3109/14767058.2014.958461.

14 Gyamfi-Bannerman C, Thom EA, Blackwell SC, et al. NICHD Maternal-Fetal Medicine Units Network. Antenatal betamethasone for women at risk for late preterm delivery. N Engl J Med 2016;374:1311-20. doi:10.1056/NEJMoa1516783.

15 Balci O, Ozdemir S, Mahmoud AS, Acar A, Colakoglu MC. The effect of antenatal steroids on fetal lung maturation between the $34^{\text {th }}$ and $36^{\text {th }}$ week of pregnancy. Gynecol Obstet Invest 2010;70:95-9. doi:10.1159/000295898

16 Nada AM, Shafeek MM, El Maraghy MA, Nageeb AH, Salah El Din AS, Awad $\mathrm{MH}$. Antenatal corticosteroid administration before elective caesarean section at term to prevent neonatal respiratory morbidity: a randomized controlled trial. Eur J Obstet Gynecol Reprod Biol 2016;199:88-91. doi:10.1016/j.ejogrb.2016.01.026

17 Stutchfield PR, Whitaker R, Gliddon AE, Hobson L, Kotecha S, Doull II. Behavioural, educational and respiratory outcomes of antenatal betamethasone for term caesarean section (ASTECS trial). Arch Dis Child Fetal Neonatal Ed 2013;98:F195-200. doi:10.1136 archdischild-2012-303157.
18 Sotiriadis A, Makrydimas G, Papatheodorou S, Ioannidis JP. Corticosteroids for preventing neonatal respiratory morbidity after elective caesarean section at term. Cochrane Database Syst Rev 2009; 4:CD006614.

19 Brownfoot FC, Gagliardi DI, Bain E, Middleton P, Crowther CA. Different corticosteroids and regimens for accelerating fetal lung maturation for women at risk of preterm birth. Cochrane Database Syst Rev 2013;8:CD006764

20 Prefumo F, Ferrazzi E, Di Tommaso M, et al. Neonatal morbidity after cesarean section before labor at 34(+0) to 38(+6) weeks: a cohort study. J Matern Fetal Neonatal Med 2016;29:1334-8. doi:10.3109/14767058.2015.1047758

21 Zanardo V, Simbi AK, Franzoi M, Soldà G, Salvadori A, Trevisanuto D. Neonatal respiratory morbidity risk and mode of delivery at term: influence of timing of elective caesarean delivery. Acta Paediatr 2004;93:643-7. doi:10.1111/j.1651-2227.2004.tb02990.x.

22 Berger PJ, Smolich J], Ramsden CA, Walker AM. Effect of lung liquid volume on respiratory performance after caesarean delivery in the lamb. J Physiol 1996;492:905-12. doi:10.1113/jphysiol.1996. sp021356.

23 Irestedt L, Lagercrantz H, Belfrage P. Causes and consequences of maternal and fetal sympathoadrenal activation during parturition. Acta Obstet Gynecol Scand Suppl 1984;118:111-5. doi:10.3109/00016348409157136.

24 Riley CA, Boozer K, King TL. Antenatal corticosteroids at the beginning of the $21^{\text {st }}$ century. J Midwifery Womens Health 2011;56:591-7. doi:10.1111/j.1542-2011.2011.00119.x.

25 Jain L, Eaton DC. Physiology of fetal lung fluid clearance and the effect of labor. Semin Perinatol 2006;30:34-43. doi:10.1053/j. semperi.2006.01.006.

26 Bloomfield T. Caesarean section, NICE Guidelines and management of labour. J Obstet Gynaecol 2004;24:485-90. doi:10.1080/0144361042331271052.

27 American College of Obstetricians and Gynecologists. ACOG committee opinion no. 561: Nonmedically indicated early-term deliveries. Obstet Gynecol 2013;121:911-5. doi:10.1097/01. AOG.0000428649.57622.a7.

28 Berghella V, Blackwell SC, Ramin SM, Sibai BM, Saade GR. Use and misuse of the term "elective" in obstetrics. Obstet Gynecol 2011;117:372-6. doi:10.1097/AOG.0b013e31820780ff.

29 Warshak CR, Ramos GA, Eskander R, et al. Effect of predelivery diagnosis in 99 consecutive cases of placenta accreta. Obstet Gynecol 2010;115:65-9. doi:10.1097/AOG.0b013e3181c4f12a.

30 Ceriani Cernadas IM. [Late-preterm infants, a growing challenge in both the short and long term]. Arch Argent Pediatr 2015;113:482-4.

31 Duvanel CB, Fawer CL, Cotting J, Hohlfeld P, Matthieu JM. Long-term effects of neonatal hypoglycemia on brain growth and psychomotor development in small-for-gestational-age preterm infants. J Pediatr 1999;134:492-8. doi:10.1016/S0022-3476(99) 70209-X.

C BMJ Publishing Group Ltd 2016

Supplementary file S1: Full electronic search from Medline

Supplementary file S2: Risk of bias table 